\title{
GLOBAL POINTWISE A PRIORI BOUNDS AND LARGE TIME BEHAVIOUR FOR A NONLINEAR SYSTEM DESCRIBING THE SPREAD OF INFECTIOUS DISEASE
}

Abstract. This paper considers a reaction-diffusion system with biatic diffusion. Existence of a globally bounded solution is proved and its large time behaviour is given.

1. Introduction. Our purpose is to analyse the following quasilinear system of reaction-diffusion equations:

$$
\begin{aligned}
& \left.\begin{array}{l}
\partial u(t, x) / \partial t=\nabla \cdot(a(u) \nabla u)-u h(u) v \\
\partial v(t, x) / \partial t=\nabla \cdot(b(v) \nabla v)+u h(u) v-\lambda v
\end{array}\right\} \quad \text { in }(0, \infty) \times \Omega, \\
& u(0, x)=\varphi(x), \quad v(0, x)=\psi(x), \quad x \in \Omega \subset \mathbb{R}^{n}
\end{aligned}
$$

( $n=1,2$ or 3 in practice), considered with the Neumann or Dirichlet boundary conditions:

$$
\partial u(t, x) / \partial \nu=\partial v(t, x) / \partial \nu=0 \quad \text { on }(0, \infty) \times \partial \Omega
$$

or

$$
u(t, x)=v(t, x)=0 \quad \text { on }(0, \infty) \times \partial \Omega
$$

where $\Omega$ is a bounded smooth domain $\left(\partial \Omega \in C^{2+\beta}\right.$ with some $\beta \in(0,1)$ ), $t \geq 0$ and $\nu$ denotes the outward normal vector to $\partial \Omega$.

The diffusivities $a(\cdot)$ and $b(\cdot)$ are assumed to be smooth, nondegenerate, strictly positive, and uniformly bounded. Namely, there are positive constants $C_{0}$ and $C_{1}$ such that

$$
0<C_{0} \leq \min (a(u), b(u))<\max (a(u), b(u)) \leq C_{1}<\infty \quad \text { for all } u \in \mathbb{R} .
$$

Also it is assumed that $h \in C^{1}\left(\mathbb{R}, \mathbb{R}^{+}\right)$and $\lambda>0$.

1991 Mathematics Subject Classification: 35K57, 35B40, 35G20.

Key words and phrases: nonlinear reaction-diffusion system, global existence, large time behaviour. 
The main motivation for studying the system (1)-(2) comes from the well known Kermack-McKendrick system which represents a basic model for the description of epidemics obeying the susceptibles-infectives-removed (S-I-R) scheme [2]:

$$
\left\{\begin{array}{l}
S^{\prime}(t)=\mu S(t) I(t), \\
I^{\prime}(t)=\mu S(t) I(t)-\lambda I(t) \quad \text { for } t \geq 0, \mu, \lambda>0, \\
R^{\prime}(t)=\lambda I(t),
\end{array}\right.
$$

where $S(t), I(t)$ and $R(t)$ are the number of susceptibles (individuals not infected but capable of becoming infected), the number of infectives (individuals capable of transmitting the disease to members of the susceptibles class), and the number of removed (individuals who have died or who have recovered and have permanent immunity), respectively.

The proposed model (1)-(2) is clearly an extension of (5). In (1)-(2), $u$ and $v$ denote respectively the density of susceptible individuals, and the density of the infective individuals at time $t \geq 0$, and at point $x$ of the habitat $\Omega$. When $a$ and $b$ are constants, the system (1)-(2) was studied by Capasso [3] and by Haraux and Kirane [4].

2. Global existence. In the sequel, arbitrary initial functions $\varphi, \psi \in$ $C^{2+\beta}(\bar{\Omega})$ satisfying suitable compatibility conditions:

(c) $\partial \varphi / \partial \nu=\partial \psi / \partial \nu=0$ on $\partial \Omega$ in the case of the Neumann conditions $\left(4^{\prime}\right)$, or $\nabla \bullet(a(\varphi) \nabla \varphi)=\varphi h(\varphi) \psi, \nabla \bullet(b(\psi) \nabla \psi)=-\varphi h(\varphi) \psi+\lambda \psi$ and $\varphi=\psi=0$ on $\partial \Omega$ in the case of the Dirichlet conditions $\left(4^{\prime \prime}\right)$

will be considered.

Now, a priori $L^{\infty}(\Omega)$-bounds are derived following the iterative method of Moser as in [1].

THEOREM. For arbitrary initial functions $\varphi, \psi \in C^{2+\beta}(\bar{\Omega}), \varphi \geq 0, \psi \geq 0$, satisfying the compatibility condition (c), there exists a unique, nonnegative, globally bounded solution $(u, v), u, v \in C^{1+\alpha / 4,2+\alpha / 2}([0, \infty) \times \bar{\Omega})$ to $(1)-(4)$ $(\alpha=\min (\beta, 1 / 2))$. In particular, the orbit $\Gamma\left(u_{0}, v_{0}\right)=\{(u(t, \cdot), v(t, \cdot))$ : $t \geq 0\}$ is relatively compact in $C^{2}\left(\bar{\Omega}, \mathbb{R}^{2}\right)$.

Proof. For fixed $u$ and $v$, the operators $\nabla_{\bullet}(a(u) \nabla u)$ and $\nabla_{\bullet}(b(v) \nabla v)$ generate analytic semigroups on $L^{p}(\Omega)$, so the system $(1)-(4)$ has a unique, noncontinuable, classical solution $(u, v)$ on $\left[0, T_{\max }\right) \times \bar{\Omega}$ for some $T_{\max }<\infty$. Moreover, $u, v \in L^{\infty}([0, T) \times \bar{\Omega})$ for $0<T<T_{\max }$ and

$$
\lim _{t \uparrow T_{\max }}\left\{T_{\max }+\|u(t, \cdot)\|_{\infty}+\|v(t, \cdot)\|_{\infty}\right\}=\infty .
$$


Now we derive a priori bounds for $(u, v)$ in $L^{\infty}(\Omega)$. Multiplying (1) by $p u^{p-1}, p$ integer, and integrating over $\Omega$, we get

(6) $\frac{d}{d t}\left(\int_{\Omega} u^{p}\right)+p(p-1) \int_{\Omega} a(u)|\nabla u|^{2} u^{p-2}=-p \int_{\Omega} h(u) u^{p} v \leq 0$,

from which we infer, for all $1 \leq p \leq \infty$,

$$
\|u(t)\|_{p} \leq\|\varphi\|_{p} \leq C,
$$

where $\|\cdot\|_{p}$ is the usual norm in $L^{p}(\Omega)$.

On the other hand,

$$
\frac{d}{d t}\left(\int_{\Omega} v^{p}\right)+p(p-1) \int_{\Omega} b(v)|\nabla v|^{2} v^{p-2}=p \int_{\Omega}\left(h(u) u v^{p}-\lambda v^{p}\right) .
$$

Now using the hypothesis on $b(\cdot)$ and the estimate $(7)$ with $p=\infty$, we obtain

$$
\frac{d}{d t}\left(\int_{\Omega} v^{p}\right)+4 C_{0} \int_{\Omega}\left|\nabla v^{p / 2}\right|^{2} \leq M p \int_{\Omega} v^{p},
$$

where $M=\|u h(u)\|_{\infty}$.

Now, fix $\theta$ with $n(n+2)^{-1}<\theta<1$ arbitrarily. Then by the GagliardoNirenberg inequality

$$
\|f\|_{2}^{2} \leq C\|f\|_{1, p}^{2 \theta}\|f\|_{1}^{2(1-\theta)} \leq \varepsilon\|f\|_{1}^{2}+C \varepsilon^{\theta(\theta-1)^{-1}}\|f\|_{1}^{2},
$$

for $\varepsilon>0$ and $f \in W^{1, p}(\Omega)$ with norm $\|f\|_{1, p}$. Thus,

$$
\|\nabla f\|_{2}^{2} \geq(1-\varepsilon) \varepsilon^{-1}\|f\|_{2}^{2}+C \varepsilon^{(\theta-1)^{-1}}\|f\|_{1}^{2}
$$

for $0<\varepsilon<1$. By inserting this inequality with $f=v^{p / 2}$ in (9) we obtain

$$
\begin{aligned}
(d / d t)\left(p^{-1}\left\|v^{p / 2}\right\|_{2}^{2}\right)+4 p^{-1} C_{0}(1-\varepsilon) \varepsilon^{-1}\left\|v^{p / 2}\right\|_{2}^{2} & \\
& -4 C_{0} C \varepsilon^{(\theta-1)^{-1}}\left\|v^{p / 2}\right\|_{1}^{2} \leq M p\left\|v^{p / 2}\right\|_{2}^{2} .
\end{aligned}
$$

Choosing $\varepsilon=4 C_{0} /\left(M p+4 C+4 C_{0}\right)$, we infer

$$
(d / d t)\left(\left\|v^{p / 2}\right\|_{2}^{2}\right) \leq 4 C\left(-\left\|v^{p / 2}\right\|_{2}^{2}+C_{0}\left\|v^{p / 2}\right\|_{1}^{2}\right) .
$$

We thus have $(d / d t)\left(\left\|v^{p / 2}\right\|_{2}^{2}\right) \leq 0$ whenever $\left\|v^{p / 2}\right\|_{2}^{2} \geq C_{0}\left\|v^{p / 2}\right\|_{1}^{2}$, and so $\left\|v^{p / 2}\right\|_{2}^{2} \leq\left\|\psi^{p / 2}\right\|_{2}^{2}$. Hence

$$
\left\|v^{p}\right\|_{1} \leq \max \left(\left\|\psi^{p}\right\|_{1}, C_{0}\left\|v^{p / 2}\right\|_{1}^{2}\right) .
$$

Now, let $M(k ; t)=\max \|v(t)\|_{l}\left(l=2^{k}\right)$ for $t \in I=\left[0, T_{\max }\right), k=$ $0,1,2, \ldots$ Then, letting $p=l$ in (10) and taking the $l$-root, it follows that

$$
M(k ; t) \leq \max (M(k ; 0), M(k-1 ; t)) .
$$

Since $\|\cdot\|_{l} \rightarrow\|\cdot\|_{\infty}$ as $k \rightarrow \infty$, we see that $M(k ; 0) \leq C$ for all $k \geq 0$, and thus $M(k ; t) \leq \max (C, M(k-1 ; t))$, from which we infer

$$
M(k ; t) \leq \max (C, M(0 ; t)) .
$$


As $(d / d t)\left(\int_{\Omega} u+v\right)=-\lambda \int_{\Omega} v \leq 0$, it follows that $\max _{t \in I}\|v\|_{1} \leq C$, and hence $M(k ; t) \leq C$. Letting $k \rightarrow \infty$ shows that for any $t \in I$,

$$
\|v(t)\|_{\infty} \leq C \text {. }
$$

The a priori estimates (7) and (11) lead to the existence of a global and bounded solution to (1)-(4). The positivity of $(u, v)$ whenever $\varphi \geq 0$ and $\psi \geq 0$ is a consequence of the Gronwall lemma.

3. Large time behaviour of the time-dependent solutions. First of all, we show that the trajectories are relatively compact in $C^{2}(\bar{\Omega})$. For the functions $u$ and $v$ solve the equations

$$
\begin{gathered}
u^{\prime}=A(t) u+f(u, v) \\
v^{\prime}=B(t) v+g(u, v) \\
\begin{array}{c}
u(0)=\varphi, \quad v(0)=\psi,
\end{array} \quad \text { for } t>0,
\end{gathered}
$$

with operators $A(t), B(t): D(A) \subset L^{p}(\Omega) \rightarrow L^{p}(\Omega)$ for $t>0$, given by

$$
D=D(A)=D(B)=\left\{w \in W^{2, p}(\Omega): \partial w / \partial \nu=0 \text { on } \partial \Omega\right\}
$$

for Neumann boundary conditions, and

$$
D=D(A)=D(B)=\left\{w \in W^{2, p}(\Omega) \cap W^{1, p}(\Omega): w=0 \text { on } \partial \Omega\right\}
$$

for Dirichlet boundary conditions,

$$
\begin{aligned}
& A(t) w(x)=\nabla_{\bullet}\left(a(w(t, x)) \nabla_{w}(t, x)\right)-k w(t, x) \quad \text { for } w \in D, \\
& B(t) w(x)=\nabla_{\bullet}\left(b(w(t, x)) \nabla_{w}(t, x)\right)-k w(t, x) \quad \text { for } w \in D,
\end{aligned}
$$

where $k$ is a constant, and

$$
f(u, v)=-h(u) u v+k u, \quad g(u, v)=h(u) u v-\lambda u+k v .
$$

For any $t \geq 0$, the operators $A(t)$ and $B(t)$ generate analytic semigroups in $L^{p}(\Omega)$ and the families $(A(t))$ and $(B(t))$ generate evolution systems $(U(t, s): 0 \leq s \leq t<\infty)$ and $(V(t, s): 0 \leq s \leq t<\infty)$ in $L^{p}(\Omega)$, respectively. Moreover, if $k>0$ is chosen sufficiently large, then there are constants $C$ and $\sigma>0$ such that

$$
\begin{gathered}
\left\|A^{\alpha}(t) A^{-\beta}(s)\right\| \leq C(\alpha, \beta) \quad \text { for all } t, s \geq 0, \\
\left\|A^{\alpha}(t) U(t, s) A^{-\beta}(s)\right\| \leq C(t-s)^{\beta-\alpha} e^{-\sigma(t-s)}
\end{gathered}
$$

for all $0 \leq s \leq t<\infty, 0 \leq \alpha<1$, and some $\sigma>0$, with constant $C$ independent of $t$ and $s$.

Solutions to (12) and (13) may be represented in the forms

$$
u(t)=U(t, 0) \varphi+\int_{0}^{t} U(t, s)[f(u(s), v(s))] d s,
$$




$$
v(t)=V(t, 0) \psi+\int_{0}^{t} V(t, s)[g(u(s), v(s))] d s .
$$

By (16) and (17) we may write

$$
\begin{aligned}
& A^{\alpha}(0) u(t)=A^{\alpha}(0) U(t, 0) \varphi+\int_{0}^{t} A^{\alpha}(0) U(t, s)[f(u(s), v(s))] d s, \\
& B^{\alpha}(0) v(t)=B^{\alpha}(0) V(t, 0) \psi+\int_{0}^{t} B^{\alpha}(0) V(t, s)[g(u(s), v(s))] d s .
\end{aligned}
$$

Now we have the estimate

$$
\text { (18) }\|u(t)\|_{\alpha} \leq\left\|A^{\alpha}(0) U(t, 0) \varphi\right\|_{p}+\int_{0}^{t}\left\|A^{\alpha}(0) U(t, s)[f(u(s), v(s))]\right\|_{p} d s
$$

where $\|\cdot\|_{0, \alpha}=\left\|A^{\alpha}(0) \cdot\right\|_{p}$.

The first term of the right-hand side of the above inequality can be estimated as follows:

(19) $\left\|A^{\alpha}(0) U(t, 0) \varphi\right\|_{p}=\left\|A^{\alpha}(0) A^{-1}(t) A(t) U(t, 0) A^{-1}(0) A(0) \varphi\right\|_{p}$

$$
\begin{aligned}
& \leq\left\|A^{\alpha}(0) A^{-1}(t)\right\|\left\|A(t) U(t, 0) A^{-1}(0)\right\|\|\varphi\|_{p} \\
& \leq C\|\varphi\|_{p} \quad(\text { by }(14),(15)) .
\end{aligned}
$$

Also

$$
\begin{aligned}
\left\|A^{\alpha}(0) U(t, s)\right\| & =\left\|A^{\alpha}(0) A^{-\alpha}(t) A^{\alpha}(t) U(t, s)\right\| \\
& \leq\left\|A^{\alpha}(0) A^{-\alpha}(t)\right\|\left\|A^{\alpha}(t) U(t, s)\right\| \\
& \leq C(t-s)^{-\alpha} e^{-\sigma(t-s)} .
\end{aligned}
$$

Hence, using (18)-(20) yields

$$
\|u(t)\|_{0, \alpha} \leq C+C \int_{0}^{t}(t-s)^{-\alpha} e^{-\sigma(t-s)} d s,
$$

so that

$$
\|u(t)\|_{0, \alpha} \leq C \quad \text { for all } t>0 .
$$

Using the same analysis, we can obtain

$$
\|v(t)\|_{0, \alpha} \leq C \quad \text { for all } t>0 .
$$

Now, using a result of Redlinger [5, Theorem 5, p. 144] we obtain

$$
u^{\prime}(t), v^{\prime}(t) \in A^{\alpha}(0)\left(L^{p}(\Omega)\right),
$$

and, for any $\delta>0$,

$$
\left\|u^{\prime}(t)\right\|_{0, \alpha},\left\|v^{\prime}(t)\right\|_{0, \alpha} \leq C(\alpha, \delta) \quad \text { for all } t>\delta,
$$

with constant $C(\alpha, \delta)$ independent of $t$. 
Now taking $p>n$ we have $D\left(A^{\alpha}\right) \subset C^{\varrho}(\Omega)(\varrho=1-n / p)$. Hence, writing (1)-(2) as an uncoupled system of linear elliptic equations $(t \geq 0)$,

$$
\begin{array}{ll}
-\nabla \cdot(\bar{a}(t, x) \nabla u)=\bar{f}(t, x)-\partial u(t, x) / \partial t & \text { in } \Omega, \\
u=0 \quad \text { or } \quad \partial u / \partial \nu=0 & \text { on } \partial \Omega,
\end{array}
$$

where

$$
\bar{a}(t, x)=a(u(t, x)), \quad \bar{f}(t, x)=-u(t, x) h(u(t, x)) v(t, x),
$$

and

where

$$
\begin{array}{ll}
-\nabla \cdot(\bar{b}(t, x) \nabla v)=\bar{g}(t, x)-\partial v(t, x) / \partial t & \text { in } \Omega, \\
v=0 \quad \text { or } \quad \partial v / \partial \nu=0 & \text { on } \partial \Omega,
\end{array}
$$

$$
\begin{aligned}
& \bar{b}(t, x)=b(v(t, x)), \\
& \bar{g}(t, x)=u(t, x) h(u(t, x)) v(t, x)-\lambda v(t, x) .
\end{aligned}
$$

Now, it follows from the Schauder estimates that

$$
u(t, \cdot), v(t, \cdot) \in C^{2+\varrho}(\Omega) \quad \text { for all } t \geq \delta .
$$

This result enables us to treat the large time behaviour question in the same manner as in our previous work with Haraux [4]. The results are:

Case of the Dirichlet boundary conditions

TheOrem 3.1. For all $t>0$ and $1 \leq p \leq \infty$,

(i) $\|u(t)\|_{p} \leq C e^{-\lambda_{p} t}\|\varphi\|_{p}$,

(ii) $\|v(t)\|_{p} \leq C e^{-\tilde{\lambda}_{p} t}\|\psi\|_{p}$,

where $\lambda_{p}, \widetilde{\lambda}_{p}>0$ for $p<\infty$ and $\lambda_{\infty}=\widetilde{\lambda}_{\infty}=0$.

Proof. We can multiply the $u$-equation by $p u^{p-1}$, integrate over $Q_{t}=$ $(0, t) \times \Omega$, and obtain

$$
\begin{aligned}
\int_{\Omega} u^{p} & \leq \int_{\Omega} \varphi^{p}-C_{0} p(p-1) \int_{Q_{t}} u^{p-2}|\nabla u|^{2} \\
& =\int_{\Omega} \varphi^{p}-\frac{4 C_{0}(p-1)}{p} \int_{Q_{t}}\left|\nabla u^{p / 2}\right|^{2} \leq \int_{\Omega} \varphi^{p}-C \int_{Q_{t}} u^{p}
\end{aligned}
$$

by the Poincaré inequality, and $C$ is independent of $p$. Now (i) follows by Gronwall's lemma where $\lambda_{p}=C / p$; (ii) can be obtained by the same scheme.

Case of the Neumann boundary conditions

Proposition. Let $(u, v)$ be the solution of $(1)-(3)$ with $\left(4^{\prime}\right)$. Then there exists a constant $C$ such that for all $t>0$,

$$
\|\nabla u\|_{2} \leq C \quad \text { and } \quad\|\nabla v\|_{2} \leq C .
$$


P r o of. As the two proofs are similar, we only give the one concerning $u$.

To begin with, integrating (1) over $Q_{t}$ yields

$$
\|u(t)\|_{1}+\int_{Q_{t}} u h(u) v d x d t=\|\varphi\|_{1}
$$

hence

$$
\int_{Q_{t}} u h(u) v d x d t \leq C .
$$

Now, multiplying (1) by $a(u) u_{t}$ and integrating over $Q_{t}$ we obtain

$$
\begin{aligned}
\int_{Q_{t}} a(u) u_{t}^{2} d x d t & +\int_{\Omega}|a(u) \nabla u|^{2} d x \\
& \leq \int_{Q_{t}}\left|a(u) u_{t}\right| u h(u) v d x d t+\int_{\Omega}|a(\varphi) \nabla \varphi|^{2} d x
\end{aligned}
$$

Applying the Young inequality yields

$$
\left|a(u) u_{t}\right| u h(u) v \leq \varepsilon\left|a(u) u_{t}\right|^{2}+(1 / 4 \varepsilon)|u h(u) v|^{2} \quad \text { for } \varepsilon>0 .
$$

Taking $\varepsilon C \leq 1$ and using (7), (11) and (21) yields

$$
\|\nabla u\|_{2} \leq C \text {. }
$$

Now, integrating (1) over $\Omega$, we see that the function $t \rightarrow \int_{\Omega} u(t, x) d x$ is decreasing, and hence goes to a limit as $t$ goes to infinity:

$$
\lim _{t \rightarrow \infty} \mu \int_{\Omega} u(t, x) d x=\bar{u}
$$

where $\mu=(\operatorname{meas}(\Omega))^{-1}$.

Also adding the left-hand side and the right-hand side of (1) and (2), then integrating the results over $\Omega$ and using the Gauss theorem to transform the terms $\nabla_{\bullet}(a(u) \nabla u)$ and $\nabla_{\bullet}(b(v) \nabla v)$ in the light of the boundary conditions we obtain

$$
\frac{d}{d t}\left(\int_{\Omega}(u(t, x)+v(t, x)) d x\right)+\lambda \int_{\Omega} v(t, x) d x=0
$$

from which we deduce that $t \rightarrow \int_{\Omega}(u+v) d x$ is decreasing, hence goes to a limit as $t \rightarrow \infty$. Combining this result with (23) leads to

$$
\lim _{t \rightarrow \infty} \int_{\Omega} v(t, x) d x=l \geq 0 .
$$

Integrating $(24)$ over $(0, t)$ yields

$$
\lambda \int_{Q_{t}} v(t, x) d x d t \leq \int_{\Omega}(\varphi+\psi) d x \quad \text { for any } t \geq 0 .
$$


From (25) and (26) we deduce that $l=0$, so

$$
\lim _{t \rightarrow \infty} \int_{\Omega} v(t, x) d x=0 .
$$

On the other hand, since $\bigcup_{t \geq 0}\{u(t)\}$ and $\bigcup_{t \geq 0}\{v(t)\}$ are precompact in $C^{2}(\bar{\Omega})$, there exists a sequence $\left\{t_{n}\right\}_{n \geq 0}$ with $t_{n} \rightarrow \infty$ such that

$$
\lim _{n \rightarrow \infty} u\left(t_{n}\right)=u^{*} \quad \text { and } \quad \lim _{n \rightarrow \infty} v\left(t_{n}\right)=v^{*} \quad \text { in } C^{2}(\bar{\Omega}),
$$

and since $v \geq 0$, we then deduce $\lim _{n \rightarrow \infty} v\left(t_{n}\right)=0$ in $C^{2}(\bar{\Omega})$.

As a consequence of (22), we have

$$
\int_{Q_{t}}\left|u_{t}\right|^{2} d x d t \leq C .
$$

In the same manner, we can get

$$
\int_{Q_{t}}\left|v_{t}\right|^{2} d x d t \leq C
$$

Therefore there exists a sequence $t_{k} \rightarrow \infty$ for which $\lim _{k \rightarrow \infty} u_{t}\left(t_{k}, x\right)=0$ a.e. on $\Omega$ or in $L^{2}(\Omega)$, and the same is true for $v_{t}\left(t_{k}, x\right)$.

Since $\lim _{k \rightarrow \infty} u\left(t_{k}\right)=u^{*}, \lim _{k \rightarrow \infty} v\left(t_{k}\right)=0$ in $C^{2}(\bar{\Omega})$, we have

$$
\begin{gathered}
u\left(t_{k}, x\right) h\left(u\left(t_{k}, x\right)\right) v\left(t_{k}, x\right) \rightarrow 0 \quad \text { as } t_{k} \rightarrow \infty, \\
u\left(t_{k}, x\right) h\left(u\left(t_{k}, x\right)\right) v\left(t_{k}, x\right)-\lambda v\left(t_{k}, x\right) \rightarrow 0 \quad \text { as } t_{k} \rightarrow \infty,
\end{gathered}
$$

uniformly in $\Omega$. Passing to the limit as $t \rightarrow \infty$ through the sequence $t_{k}$ in (1) in the sense of $L^{2}(\Omega)$ and using the closedness of the operator $\nabla_{\bullet}\left(a(\cdot) \nabla_{\bullet}\right)$ in $L^{2}(\Omega)$, we find the equation

$$
-\nabla\left(a\left(u^{*}\right) \nabla u^{*}\right)=0 \quad \text { in } L^{2}(\Omega) ;
$$

furthermore,

$$
\partial u^{*} / \partial \nu=0 \quad \text { on } \partial \Omega
$$

Now, solving equation (27) subject to the constraint (28) yields $u^{*}=m$, and by $(23)$ we infer that $m \equiv \bar{u}$.

We can check as in [4] that if $\varphi \not \equiv 0$ then $\bar{u}>0$.

Acknowledgments. The author would like to thank Professor Abdus Salam, the International Atomic Energy Agency and UNESCO for hospitality at the International Centre for Theoretical Physics, Trieste.

\section{References}

[1] N. D. Alikakos, $L^{p}$ bounds of solutions of reaction diffusion equations, Comm. Partial Differential Equations 4 (1979), 827-869. 
[2] N. T. J. Bailey, The Mathematical Theory of Infectious Diseases, 2nd ed., Griffin, London, 1975.

[3] V. Capasso, Global solution for a diffusive nonlinear deterministic epidemic model, SIAM J. Appl. Math. 35 (1978), 274-284.

[4] A. Haraux et M. Kirane, Estimations $C^{1}$ pour des problèmes paraboliques semilinéaires, Ann. Fac. Sci. Toulouse 5 (1983), 265-280.

[5] R. Redlinger, Compactness results for time-dependent parabolic systems, J. Differential Equations 64 (1986), 133-153.

M. KIRANE

INTERNATIONAL CENTRE

FOR THEORETICAL PHYSICS

MATHEMATICS SECTION

P.O. BOX 586

I-34100 TRIESTE, ITALY
DÉPARTEMENT DE MATHÉMATIQUES UNIVERSITÉ DE ANNABA B.P. 12 ANNABA 23000, ALGERIA

Received on 11.12.1990; revised version on 12.6.1992 